\title{
Green Chemistry: Air-Triggered Catalyst- and Oxidant-Free Decarboxylative Oxysulfonylation of Arylpropiolic Acids With Sodium Sulfinates
}

\section{Xingyu Chen}

China Academy of Chinese Medical Sciences Institute of Chinese Materia Medica

\section{Xiaoqiang Chang}

China Academy of Chinese Medical Sciences Institute of Chinese Materia Medica

\section{Shuaichen Zhang}

China Academy of Chinese Medical Sciences Institute of Chinese Materia Medica

\section{Sixian Lu}

China Academy of Chinese Medical Sciences Institute of Chinese Materia Medica

\section{Lan Yang}

China Academy of Chinese Medical Sciences Institute of Chinese Materia Medica

\section{Peng Sun ( $\nabla$ psun@icmm.ac.cn )}

Institute of Chinese Materia Medica, Academy of Chinese Medical Sciences https://orcid.org/00000003-0348-5405

\section{Research Article}

Keywords: Air, Catalyst-free, Oxidant-free, Decarboxylative oxysulfonylation, $\beta$-Keto sulfones, Green chemistry, Room temperature

Posted Date: December 17th, 2021

DOI: https://doi.org/10.21203/rs.3.rs-1091233/v1

License: (c) (1) This work is licensed under a Creative Commons Attribution 4.0 International License. Read Full License 


\section{Abstract}

The exploration of novel green synthetic strategies to obtain useful organic molecules is one of the most important missions for sustainable development. Herein, an efficient and sustainable decarboxylative oxysulfonylation between arylpropiolic acids and sodium sulfinates has been established, providing a broad scope of $\beta$-ketosulfones in excellent yields. The reactions proceed at room temperature employing air as the only oxidant and oxygen source without extra catalyst, oxidant, and additive. Additionally, the reaction is scalable, and the products have been easily isolated by simple recrystallization, avoiding the chromatographic purification. Mechanistic studies have also been conducted to reveal that the reaction proceed via a radical mechanism.

\section{Introduction}

Over the last few decades, decarboxylative-coupling reaction of carboxylic acids has become an important and robust instrument in the synthetic chemist's toolkit (Goossen et al. 2008; Sharma et al. 2021; Varenikov et al. 2021; Wei et al. 2017). Owing to the potential driving force in the breaking of C$\mathrm{COOH}$ bond, such a synthetic strategy is easier to overcome the thermodynamic barriers compared with the direct $\mathrm{C}-\mathrm{H}$ activation, offering an effective route to construct various valuable carbon-carbon and carbon-heteroatom bonds with fascinating chemo- and regio-selectivity (Nanjo et al. 2018; Nanjo et al. 2019). However, these reactions are dominated by C-C (Kaur et al. 2019; Liu et al. 2016; Rodriguez et al. 2011) and C-N bond-forming transformations (Arshadi et al. 2019; Majedi et al. 2019; Wang et al. 2020). The field of decarboxylative C-S bond-forming reactions remained relatively quiet. In 2009, Duan (Duan et al. 2009) reported the first direct decarboxylative coupling between ortho-substituted aryl carboxylic acids with thiols to produce aryl sulfides. Later, a number of processes have been devised in the C-S bond construction through decarboxylative strategy (Shen et al. 2015; Hosseinian et al. 2018). In this context, most of these cases could be summarized into two patterns (Fig. 1a): (i) The cross-coupling reactions of C-M species with various sulfurative reagents after transition-metal-mediated decarboxylation of carboxylic acids; (ii) The oxidants triggered radical coupling reactions of carbon radical with sulfur radicals through the decarboxylation of carboxyl radical. However, these methods always required expensive and toxic metal catalysts, photoredox catalysts, and external oxidants, which severely limited their application in pharmaceutical and industrial manufacturing. Hence, the catalyst- and oxidant-free decarboxylative $\mathrm{C}-\mathrm{S}$ bond forming reactions are incontrovertibly in great demand but remain largely underdeveloped.

Organosulfones are important structural motifs widely distributed in natural products and bioactive agents (Dunbar et al. 2017; Scott et al. 2018; Zhao et al. 2019). These privileged moieties have also been employed as the subsequent intermediates for a range of useful organic transformations. Due to their significant applications, the preparation of organosulfones has attracted considerable attentions of synthetic and pharmaceutical chemists (Alba et al. 2010; Liu et al. 2015; Manolikakes et al. 2016; Shaaban et al. 2017). Classically, organosulfones were prepared through thioether oxidation and direct Csulfonylation processes. Recently, radical involved sulfonylation of alkynes with different sulfonyl 
precursors has emerged as an efficient solution to prepare $\beta$-keto sulfones because of its elegant atomeconomy and step-economy (Lu et al. 2013; Handa et al. 2014; Ni et al. 2020; Pampana et al. 2021). However, these processes are concomitant with the highly reactive and unstable vinyl radical intermediates (Wille 2013), making it difficult to avoid of the unwanted vinyl sulfones by-products via atom transfer radical addition (ATRA) (An et al. 2021). Moreover, the use of terminal alkynes always accompanied with undesirable side reactions such as Glaser homocoupling (Su et al. 2016; Sindhu et al. 2014). To this end, alkynyl carboxylic acids, as a kind of promising surrogate for terminal alkynes, appear to be fitted building blocks because of their superior stability, easy handling and better regio- and chemoselectivity. In 2017, Wu (Yu et al. 2017) disclosed a Cu (I)-catalyzed multicomponent reaction of aryldiazonium tetrafluoroborates, 3-arylpropiolic acids, and sulfur dioxide to construct $\beta$-ketosulfones (Fig. 1b). Later, a manganese (III)-mediated decarboxylative oxysulfonylation of arylpropiolic acids was explored by Lu (Xiong et al. 2018). Excess manganous (III) acetate were employed as catalyst and oxidant (Fig. 1C). As our unremitting effort in the antimalarial drug exploration from natural products and synthetic molecules under the guidance of Prof. Youyou Tu (Chen et al. 2020; Ma et al. 2019; Tu 2016), herein, we delivered an eco-friendly and convenient synthetic approach to $\beta$-ketosulfones for further bioevaluation (Fig. 1d). Compared to the existing methods, this protocol exhibited notable features as following: (i) Simple and sustainable conditions without toxic metal catalysts, oxidants and additives; (ii) Air was used as the only oxidant and oxygen source; (iii) Excellent regio- and chemo-selectivity without tricky by-products (e.g., Glaser-Hay homocoupling products and vinyl sulfones); (iv) Facilitative products purification avoiding column chromatography; (v) Easily to scaled up; (vii) Application in the direct synthesis and late-stage modification of complex bioactive agents.

\section{Experimental}

\section{General Information}

All the commercially available reagents were used as received. ${ }^{1} \mathrm{H}$ and ${ }^{13} \mathrm{C}$ NMR spectra were collected on BRUKER AV-600 (600 MHz) spectrometer using $\mathrm{CDCl}_{3}$ as solvent. High Resolution Mass measurement was performed on Waters Xevo G2-XS QTOF mass spectrometer with electron spray ionization (ESI) as the ion source. All the experiments were monitored by thin-layer chromatography (TLC) on commercial silica gel plates (GF254) and visualized under ultraviolet (UV) lamp at $254 \mathrm{~nm}$. Recrystallization was performed in ethyl acetate/petroleum ether (EA/PE).

\section{General procedure for the synthesis of Arylpropiolic Acids (1)}

A $10 \mathrm{~mL}$ vessel was charged with caesium carbonate $(6 \mathrm{mmol})$, silver (I) oxide (1 mol\%) and dimethyl sulfoxide (DMSO) $(5 \mathrm{~mL})$. The reaction vessel was purged with carbon dioxide, and the alkyne (5 mmol) was added via syringe. The resulting mixture was stirred for $16 \mathrm{~h}$ at $50{ }^{\circ} \mathrm{C}$ at ambient $\mathrm{CO}_{2}$ pressure. At the end of the reaction, the reaction mixture was cooled to room temperature and diluted with water. Then the aqueous layer was acidified with aqueous $\mathrm{HCl}$ and extracted with $\mathrm{EA}(3 \times 30 \mathrm{~mL})$. The combined organic 
layers were washed with brine, dried over magnesium sulfate $\left(\mathrm{MgSO}_{4}\right)$, filtered and the volatiles were removed in vacuo to afford the corresponding arylpropiolic acids 1.

\section{General procedure for the synthesis of sodium sulfinate substrates (2)}

A $25 \mathrm{~mL}$ round bottom flask was charged with sodium sulfite $(20 \mathrm{mmol})$, sodium bicarbonate $(20 \mathrm{mmol})$ and deionized $\mathrm{H}_{2} \mathrm{O}(10 \mathrm{~mL})$. After stirring for $5 \mathrm{~min}$, the sulfonyl chloride $(10 \mathrm{mmol})$ was added portionwise to the flask. The mixture was heated to $80^{\circ} \mathrm{C}$ in an oil bath for $12 \mathrm{~h}$. After cooling to room temperature, water was removed under vacuum, affording the crude sulfinate salt. Recrystallization of the residue in ethanol afforded the corresponding sodium sulfinates 2 .

\section{General procedure for the synthesis of $\beta$-keto sulfones (3)}

A mixture of aryl alkynes $1(1 \mathrm{mmol})$, sulfinates $2(10 \mathrm{mmol})$ and HFIP was stirred at $25^{\circ} \mathrm{C}$ under air atmosphere for $6 \mathrm{~h}$. Upon completion, the solvent was removed by rotary evaporation. The residue was extracted with EA $(3 \times 30 \mathrm{~mL})$. The organic phase was washed with water and brine, respectively. The solvent was concentrated in vacuo and purified by recrystallization to give the desired $\beta$-keto sulfones $\mathbf{3}$.

\section{Results And Discussion}

The research originated from the reaction of phenylpropiolic acid $\mathbf{1 a}$ with sodium benzenesulfinate $\mathbf{2 a}$. $\beta$ Ketosulfone product 3 aa was generated in 32\% yield in hexafluoroisopropanol (HFIP) at room temperature for 10 hours. Motivated by this initial result, various conditions were screened to promote the isolated yield to $93 \%$ (Table 1, Supporting Information (SI)). We then set out to investigate the generality of this method. First, a vast array of alkynyl carboxylic acids were tested (Fig. 2). Methyl, methoxyl, and phenyl substituted substrates were well-tolerated to give corresponding products in $81 \%-91 \%$ yields. Product with bromo group (3af) was prepared in 90\% yield. Electron-withdrawing groups such as cyano, trifluoromethyl, ester, and aldehyde groups were compatible with the conditions, and the corresponding products were synthesized in the yields of $73-86 \%$ (3ae, 3ag-3ai). Thienyl and naphthyl propiolic acids were also suitable substrates, and offering $3 \mathrm{am}$ and 3 an in $88 \%$ and $90 \%$ yields, respectively. Next, the scope of sodium sulfinates were evaluated. Benzenesulfinates bearing electron-donating groups such as ${ }^{t} \mathrm{Bu},-\mathrm{OMe}$, and -NHAc provided up to $90 \%$ yields (3bb-3bd). Sulfinates with moderate to strong electronwithdrawing groups (e.g., halogen, $\mathrm{OCF}_{3}, \mathrm{CN}, \mathrm{NO}_{2}$ ) remained suitable under the conditions (3be-3bi, $69 \%-85 \%)$. Naphthyl and thiophenyl-substitued counterparts took part in this transformation equally well, leading to $\mathbf{3 b j}$ and $\mathbf{3 b q}$ in the yields of $81 \%$ and $92 \%$, respectively. Further efforts were made to evaluate the alkyl sulfinates. Pleasingly, both cyclic and acyclic alkyl sulfinates delivered products in nearly quantitive yields (3bl-3bm).

To demonstrate the synthetic utility of the developed chemistry, the reaction was carried out in $10 \mathrm{mmol}$ scale, and the target product was synthesized without loss of efficiency. Following similar procedures, some representative biologically active molecules such as 3af (anti-analgesic agents) (Abdel-Aziz et al. 
2014), 3ag (11ß-hydroxysteroid dehydrogenase type I inhibitors) (Xiang et al. 2007), and 3an (carboxylesterase 1) (Han et al. 2018) were also obtained in gram scale from the corresponding arylpropiolic acids (Fig.3a). It is noteworthy that, the estrone unit, which broadly exist in drugs and bioactive molecules, could be efficiently assembled into 5 in over $90 \%$ yield (Fig.3b). This outcome highlighted the applicability and versatility of the present protocol.

Next, some experiments were carried out to probe the possible mechanism. The addition of 2,2,6,6tetramethyl-1-piperidinyloxy (TEMPO) into the reaction mixture gave no desired products (Fig. 1a., $\mathrm{SI}$ ), which indicated that the reaction might be involved in a radical pathway. Then, in the presence of diphenylethene, a sulfonylative adduct 6 was detected by HRMS (Fig. 1b., SI), inferring the generation of sulfonyl radical. Moreover, when butylated hydroxytoluene (BHT) was added to this reaction system, the desired reaction was diminished dramatically and the capture of the superoxide radical anion $\left(\mathrm{O}_{2}{ }^{--}\right)$was observed by HRMS (BHT-OOH, 7) (Fig. 1C., SI). On the other hand, the oxo-sulfonylation did not occur under nitrogen atmosphere (Fig. 1d., SI). When we studied the reaction under ${ }^{18} \mathrm{O}_{2}(97 \%)$ atmosphere, the ${ }^{18}$ O-labled ratio of the ketone 3aa was $68 \%$ (Fig. 1e., SI). Furthermore, performing the reaction in the presence of $\mathrm{H}_{2}{ }^{18} \mathrm{O}$ (10 equiv.) under the optimal conditions, only $6 \% 3$ aa was labled with ${ }^{18} \mathrm{O}$ (Fig. 1f., SI). These results indicated that the molecular oxygen was the oxidant as well as the 0 -source of the products.

Based on the aforementioned results and previous works (Chen et al. 2020; Lu et al. 2013; Lu et al. 2015), a tentative reaction pathway is depicted in Fig. 4. Initially, sodium sulfinate was activated by oxygen via autoxidation with formation of oxygen radical $\mathbf{A}$, resonating with sulfonyl radical $\mathbf{B}$, while producing superoxide radical anion $\mathrm{O}_{2}{ }^{--}$. Subsequently, the addition of $\mathbf{B}$ to alkynyl acids 1 offered vinyl radical $\mathbf{C}$, which could be further trapped by dioxygen to form the peroxy radical $\mathbf{D}$. Afterwards, intermediate $\mathbf{D}$ undergoes a single electron transfer (SET) to generate peroxide anion intermediate $\mathbf{E}$. Besides, the capture of $\mathrm{O}_{2}{ }^{--}$by the intermediate $\mathrm{C}$ may also generated the intermediate $\mathrm{E}$, which further went through intramolecular proton transfer (PT) to render the hydroperoxide intermediate $\mathbf{F}$. Finally, the reduction of intermediate $\mathbf{F}$ furnished species $\mathbf{G}$, followed by isomerization to give the desired $\beta$-keto sulfone $\mathbf{3}$.

\section{Conclusion}

In summary, we have developed a practical and green decarboxylative oxysulfonylation between arylpropiolic acids and sodium sulfinates that allows the rapid synthesis of diversely functionalized $\beta$ ketosulfones. This reaction features mild and sustainable conditions, simple operation, good functional group tolerance, and broad substrate scope. Moreover, this is the first solely dioxygen triggered decarboxylative oxysulfonylation reaction of arylpropiolic acids, which enriches the repertoire of the molecular oxygen in the decarboxylative coupling reactions toward sustainable synthesis of various valuable compounds.

\section{Abbreviations}




\begin{tabular}{ll} 
HFIP & 1,1,1,3,3,3-hexafluoro-2-propanol \\
\hline NMR & Nuclear magnetic resonance \\
\hline Q-TOF & Quadrupole time-of-flight \\
\hline UV & Ultraviolet \\
\hline PE & Petroleum ether \\
\hline CDCl $_{3}$ & Chloroform- $d$ \\
\hline equiv. & Equivalent \\
\hline TEMPO & $2,2,6,6-$ Tetramethyl-1-piperidinyloxy \\
\hline SET & Single electron transfer \\
\hline DMSO & Dimethyl sulfoxide \\
\hline ATRA & Atom transfer radical addition \\
\hline MHz & Megahertz \\
\hline ESI & Electrospray ionization \\
\hline TLC & Thin-layer chromatography \\
\hline EA & Ethyl acetate \\
\hline mmol & Millimole \\
\hline HCl & Hydrochloric acid \\
\hline BHT & Butylated hydroxytoluene \\
\hline PT & Proton transfer \\
\hline
\end{tabular}

\section{Declarations}

\section{Supporting Information}

General information, general experimental procedure, mechanism studies, characterization data for compounds and NMR spectra of compounds (PDF)

\section{Notes}

The authors declare no competing financial interest.

\section{Acknowledgment}

The author thanks the generous financial support from the Scientific and Technological Innovation Project of China Academy of Chinese Medical Sciences CI2021A05102, the National Natural Science 
Foundation of China $(21702235,81841001)$, the Fundamental Research Funds for the Central Public Welfare Research Institutes (ZZ13-YQ-098, ZZ14-FL-010, ZZ15-ND-10).

\section{References}

1. Abdel-Aziz HA, Al-Rashood KA, ElTahir K E H, Suddek GM (2014) Synthesis of $N$ benzenesulfonamide-1 H-pyrazoles bearing arylsulfonyl moiety: novel celecoxib analogs as potent anti-inflammatory agents. Eur J Med Chem 80:416-422

2. Alba AN, Companyó X, Rios R (2010) Sulfones: new reagents in organocatalysis. Chem Soc Rev 39:2018-2033

3. An S, Song KH, Lee S (2021) Vinyl sulfone synthesis via copper-catalyzed three-component decarboxylative addition. Org Biomol Chem 19:7827-7831 http://doi.org/10.1039/d1 ob01435h

4. Arshadi S, Ebrahimiasl S, Hosseinian A, Monfared A, Vessally E (2019) Recent developments in decarboxylative cross-coupling reactions between carboxylic acids and $\mathrm{N}-\mathrm{H}$ compounds. RSC Adv 9:8964-8976 http://doi.org/10.1039/c9ra00929a

5. Chen J, Allyson ZG, Xin JR, Guan Z, He YH (2020) Photo-Mediated Decarboxylative Ketonization of Atropic Acids with Sulfonyl Hydrazides: Direct Access to $\beta$-Ketosulfones. Adv Synth Catal 362:20452051 http://doi.org/10.1002/adsc.201901525

6. Chen X, Xia F, Zhao Y, Ma J, Ma Y, Zhang D, Yang L, Sun P (2020) TBHP-Mediated Oxidative Decarboxylative Cyclization in Water: Direct and Sustainable Access to Anti-malarial Polycyclic Fused Quinazolinones and Rutaecarpine. Chin J Chem 38:1239-1244 http://doi.org/10.1002/cjoc.202000154

7. Duan Z, Ranjit S, Zhang P, Liu X (2009) Synthesis of aryl sulfides by decarboxylative C-S crosscouplings. Chem Eur J 15:3666-9 http://doi.org/10.1002/chem.200900133

8. Dunbar KL, Scharf DH, Litomska A, Hertweck C (2017) Enzymatic Carbon-Sulfur Bond Formation in Natural Product Biosynthesis. Chem Rev 117:5521-5577 http://doi.org/10.1021/acs.chemrev.6b00697

9. Goossen LJ, Rodriguez N, Goossen K (2008) Carboxylic acids as substrates in homogeneous catalysis. Angew Chem Int Ed 47:3100-3120 http://doi.org/10.1002/anie.200704782

10. Han F, Su B, Song P, Wang Y, Jia L, Xun S, Hu M, Zou L (2018) N-bromosuccinimide mediated decarboxylative sulfonylation of $\beta$-keto acids with sodium sulfinates toward $\beta$-keto sulfones: Evaluation of human carboxylesterase 1 activity. Tetrahedron 74:5908-5913

11. Handa S, Fennewald JC, Lipshutz BH (2014) Aerobic oxidation in nanomicelles of aryl alkynes, in water at room temperature. Angew Chem Int Ed 53:3432-5 http://doi.org/10.1002/anie.201310634

12. Hosseinian A, Nezhad PDK, Ahmadi S, Rahmani Z, Monfared A (2018) A walk around the decarboxylative C-S cross-coupling reactions. J Sulfur Chem 40:88-112 http://doi.org/10.1080/17415993.2018.1515314 
13. Kaur P, Kumar V, Kumar R (2019) Recent advances in decarboxylative C-C bond formation using direct or in situ generated alkenyl acids. Catal Rev 62:118-161

14. Liu G, Fan C, Wu J (2015) Fixation of sulfur dioxide into small molecules. Org Biomol Chem 13:1592-9 http://doi.org/10.1039/c4ob02139h

15. Liu P, Zhang G, Sun P (2016) Transition metal-free decarboxylative alkylation reactions. Org Biomol Chem 14:10763-77 http://doi.org/10.1039/c6ob02101h

16. Lu Q, Zhang J, Zhao G, Qi Y, Wang H, Lei A (2013) Dioxygen-triggered oxidative radical reaction: direct aerobic difunctionalization of terminal alkynes toward beta-keto sulfones. J Am Chem Soc 135:11481-4 http://doi.org/10.1021/ja4052685

17. Lu Q, Zhang J, Peng P, Zhang G, Huang Z, Yi H, Miller JT, Lei A (2015) Operando X-ray absorption and EPR evidence for a single electron redox process in copper catalysis. Chem Sci 6:4851-4854 http://doi.org/10.1039/c5sc00807g

18. Ma Y, Zhu Y, Zhang D, Meng Y, Tang T, Wang K, Ma J, Wang J, Sun P (2019) Eco-friendly decarboxylative cyclization in water: practical access to the anti-malarial 4-quinolones. Green Chem 21:478-482 http://doi.org/10.1039/c8gc03570a

19. Majedi S, Majedi S, Behmagham F (2019) Recent advances in decarboxylative nitration of carboxylic acids. Chem Rev Lett 2:187-192 http://doi.org/10.22034/crl.2020.218207.1035

20. Manolikakes G, Liu N-W, Liang S (2016) Recent Advances in the Synthesis of Sulfones. Synthesis 48:1939-1973

21. Nanjo T, Kato N, Takemoto Y (2018) Oxidative Decarboxylation Enables Chemoselective, Racemization-Free Esterification: Coupling of alpha-Ketoacids and Alcohols Mediated by Hypervalent lodine (III). Org Lett 20:5766-5769 http://doi.org/10.1021/acs.orglett.8b02466

22. Nanjo T, Kato N, Zhang X, Takemoto Y (2019) A Hydroperoxide-Mediated Decarboxylation of alphaKetoacids Enables the Chemoselective Acylation of Amines. Chem Eur J 25:15504-07 http://doi.org/10.1002/chem.201904717

23. Ni B, Zhang B, Han J, Peng B, Shan Y, Niu T (2020) Heterogeneous Carbon Nitrides Photocatalysis Multicomponent Hydrosulfonylation of Alkynes to Access beta-Keto Sulfones with the Insertion of Sulfur Dioxide in Aerobic Aqueous Medium. Org Lett 22:670-674 http://doi.org/10.1021/acs.orglett.9b04454

24. Pampana VKK, Charpe VP, Sagadevan A, Das DK, Lin C-C, Hwu JR, Hwang KC (2021) Oxysulfonylation of terminal alkynes via $\mathrm{C}-\mathrm{S}$ coupling enabled by copper photoredox catalysis. Green Chem 23:3569-3574

25. Rodriguez N, Goossen LJ (2011) Decarboxylative coupling reactions: a modern strategy for C-C-bond formation. Chem Soc Rev 40:5030-5048 http://doi.org/10.1039/c1cs15093f

26. Scott KA, Njardarson JT (2018) Analysis of US FDA-Approved Drugs Containing Sulfur Atoms. Top Curr Chem (Cham) 376:5 http://doi.org/10.1007/s41061-018-0184-5

27. Shaaban S, Liang S, Liu NW, Manolikakes G (2017) Synthesis of sulfones via selective C-Hfunctionalization. Org Biomol Chem 15:1947-1955 http://doi.org/10.1039/c6ob02424f 
28. Sharma R, Yadav MR (2021) Recent developments in decarboxylative $C$ (aryl) - $X$ bond formation from (hetero) aryl carboxylic acids. Org Biomol Chem 19:5476-5500 http://doi.org/10.1039/d1ob00675d

29. Shen C, Zhang P, Sun Q, Bai S, Hor TA, Liu X (2015) Recent advances in C-S bond formation via C-H bond functionalization and decarboxylation. Chem Soc Rev 44:291-314 https://doi.org/10.1039/C4CS00239C

30. Sindhu K, Anilkumar G (2014) Recent advances and applications of Glaser coupling employing greener protocols. RSC Adv 4:27867-87 https://doi.org/10.1039/C4RA02416H

31. Su L, Dong J, Liu L, Sun M, Qiu R, Zhou Y, Yin S-F (2016) Copper catalysis for selective heterocoupling of terminal alkynes. J Am Chem Soc 138:12348-51 https://doi.org/10.1021/jacs.6b07984

32. Tu Y (2016) Artemisinin-A Gift from Traditional Chinese Medicine to the World (Nobel Lecture). Angew Chem Int Ed 55:10210-26 http://doi.org/10.1002/anie.201601967

33. Varenikov A, Shapiro E, Gandelman M (2021) Decarboxylative Halogenation of Organic Compounds. Chem Rev 121:412-484 http://doi.org/10.1021/acs.chemrev.0c00813

34. Wei Y, Hu P, Zhang M, Su W (2017) Metal-Catalyzed Decarboxylative C-H Functionalization. Chem Rev 117:8864-8907 http://doi.org/10.1021/acs.chemrev.6b00516

35. Wang Y, Tian L, Zheng Y, Shao X, Ramadoss V (2020) Recent Developments in Photochemical and Electrochemical Decarboxylative $\mathrm{C}\left(\mathrm{sp}^{3}\right)-\mathrm{N}$ Bond Formation. Synthesis 52:1357-1368 http://doi.org/10.1055/s-0039-1690839

36. Wille U (2013) Radical cascades initiated by intermolecular radical addition to alkynes and related triple bond systems. Chem Rev 113:813-853 https://doi.org/10.1021/cr100359d

37. Xiang J, Ipek M, Suri V, Tam M, Xing Y, Huang N, Zhang Y, Tobin J, Mansour TS, McKew J (2007) $\beta$ Keto sulfones as inhibitors of $11 \beta$-hydroxysteroid dehydrogenase type I and the mechanism of action. Bioorgan Med Chem 15:4396-4405

38. Xiong Y-S, Weng J, Lu G (2018) Manganese (III)-Mediated and Catalyzed Decarboxylative Hydroxysulfonylation of Arylpropiolic Acids with Sodium Sulfinates in Water. Adv Synth Catal 360:1611-1616

39. Yu J, Mao R, Wang Q, Wu J (2017) Synthesis of $\beta$-keto sulfones via a multicomponent reaction through sulfonylation and decarboxylation. Org Chem Front 4:617-621 http://doi.org/10.1039/c7qo00026j

40. Zhao C, Rakesh KP, Ravidar L, Fang WY, Qin HL (2019) Pharmaceutical and medicinal significance of sulfur (S(VI))-Containing motifs for drug discovery: A critical review. Eur J Med Chem 162:679-734 http://doi.org/10.1016/j.ejmech.2018.11.017

\section{Figures}


a) The recent $\mathrm{C}-\mathrm{S}$ bond-forming process via decarboxylative-coupling transformations.

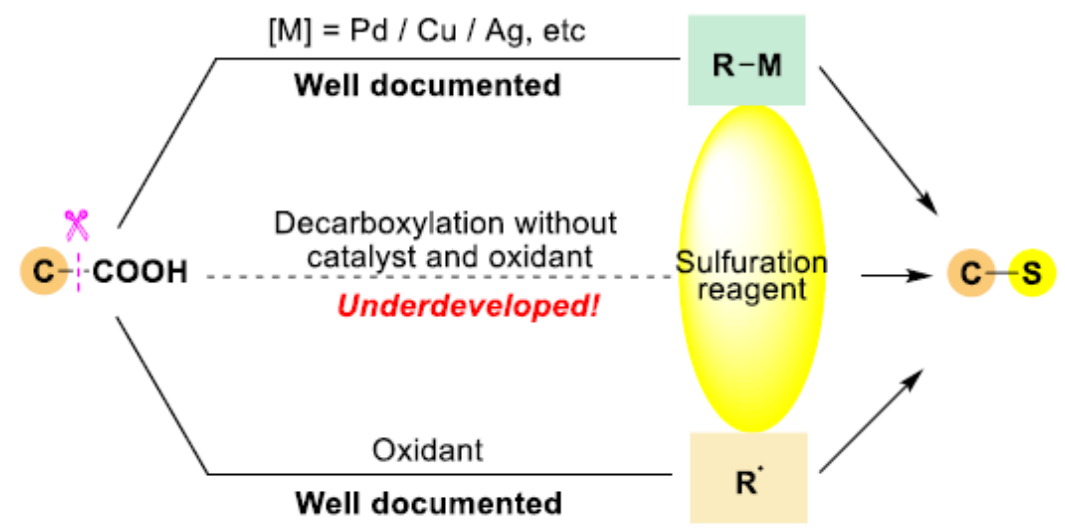

Previous work: decarboxylative oxysulfonylation of arylpropiolic acids

b) Wu's work

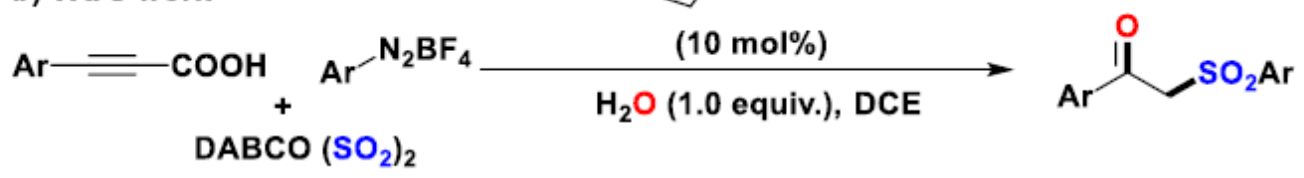

c) Lu's work

\section{Condition A}

$\mathrm{Mn}(\mathrm{OAc})_{3} \cdot 2 \mathrm{H}_{2} \mathrm{O}(3.0$ equiv.)

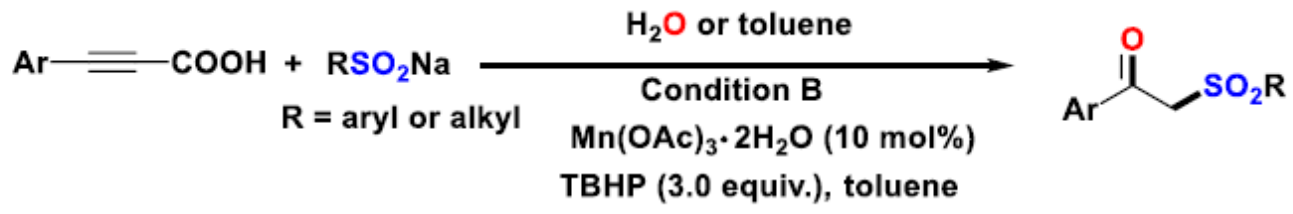

d) This work:

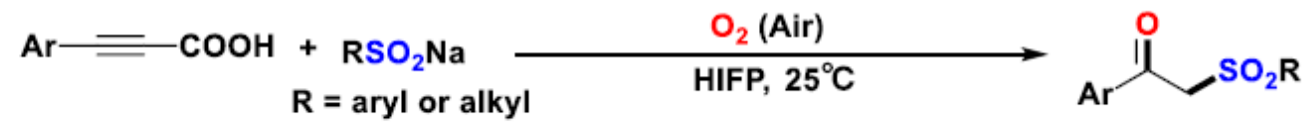

\section{Figure 1}

The recent C-S bond-forming process via decarboxylative-coupling transformations and strategies for the $\beta$-keto sulfone synthesis. 


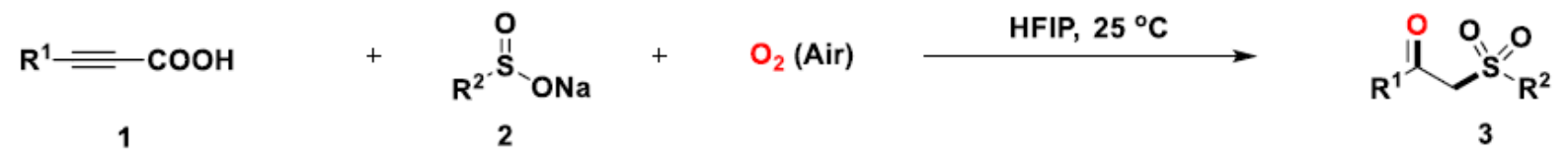

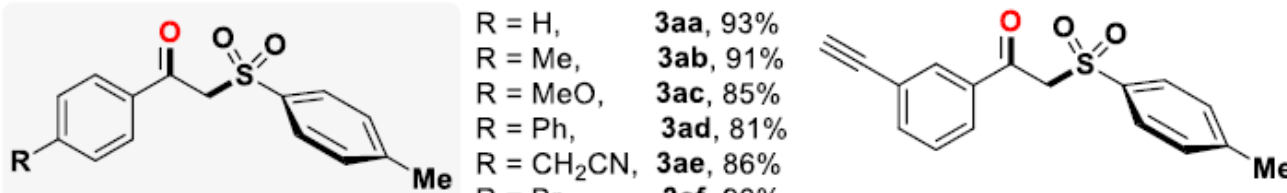

3aj, $66 \%$

$\mathrm{R}=\mathrm{CF}_{3}$

$\mathrm{R}=$ COOMe, $3 \mathrm{ah}, 76 \%$

$\mathrm{R}=\mathrm{CHO}, \quad 3 \mathrm{ai}, 73 \%$

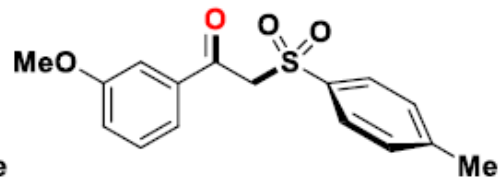

3ak, $85 \%$

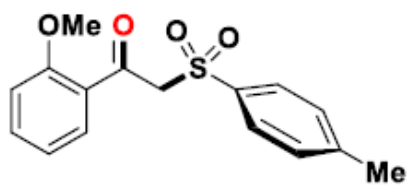

3al, $82 \%$

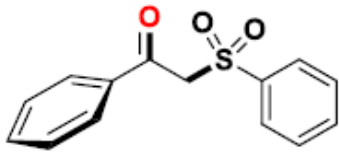

3ba, $92 \%$

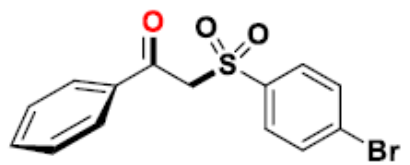

3be, $82 \%$

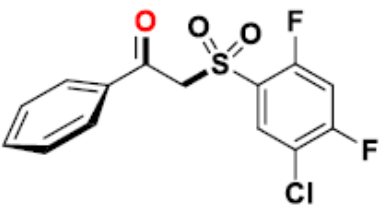

3 bi, $69 \%$

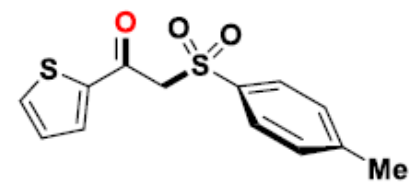

3am, 88\%

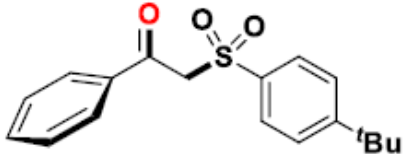

3bb, $90 \%$

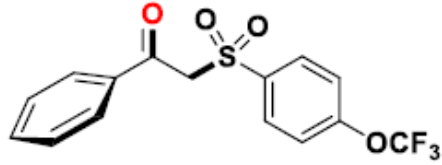

3 bf, $85 \%$

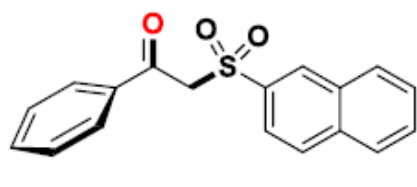

3bj, $81 \%$

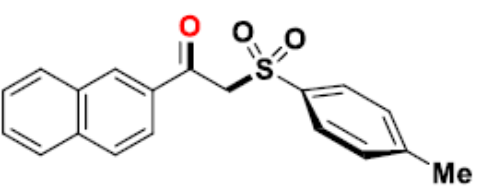

3an, 90\%<smiles>COc1ccc(S(=O)(=O)CC(=O)c2ccccc2)cc1</smiles>

3 bc, $88 \%$<smiles>N#Cc1ccc(S(=O)(=O)CC(=O)c2ccccc2)cc1</smiles>

$3 \mathrm{bg}, 73 \%$<smiles>O=C(CS(=O)(=O)c1cccs1)c1ccccc1</smiles>

3bk, 92\%

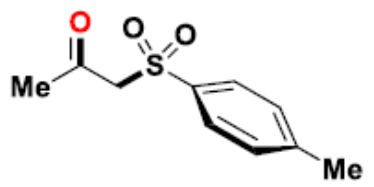

3ao, N.D

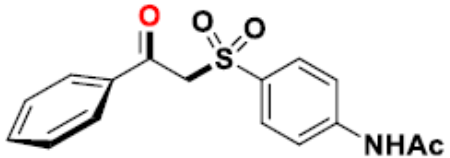

3bd, $85 \%$

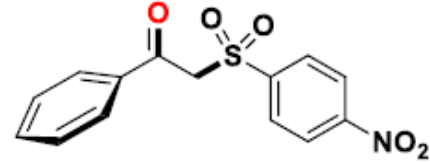

3bh, 70\%

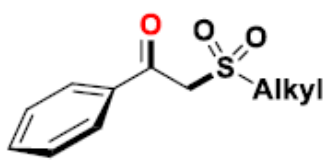

Alkyl = Cyclopropyl $3 \mathbf{b l}, 97 \%$

Alkyl = Ef 3 bm, 95\%

Figure 2

Scope of the reaction. 
(a) Pictures of the corresponding products via recrystallization:
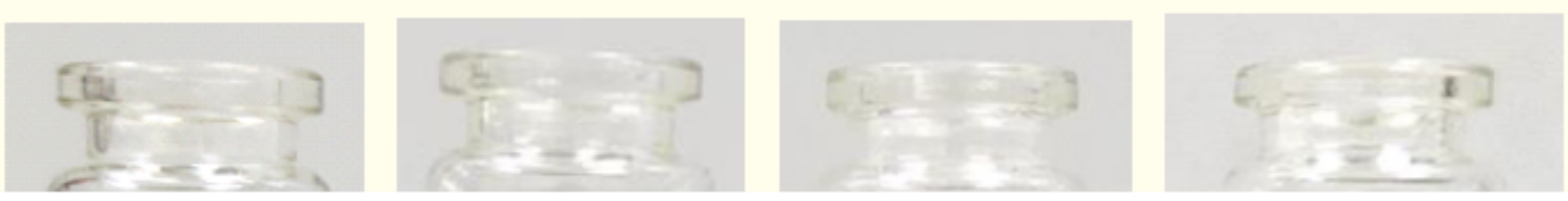

Figure 3

Gram-scale reaction and late-stage oxy-sulfonylation of bioactive molecules. 


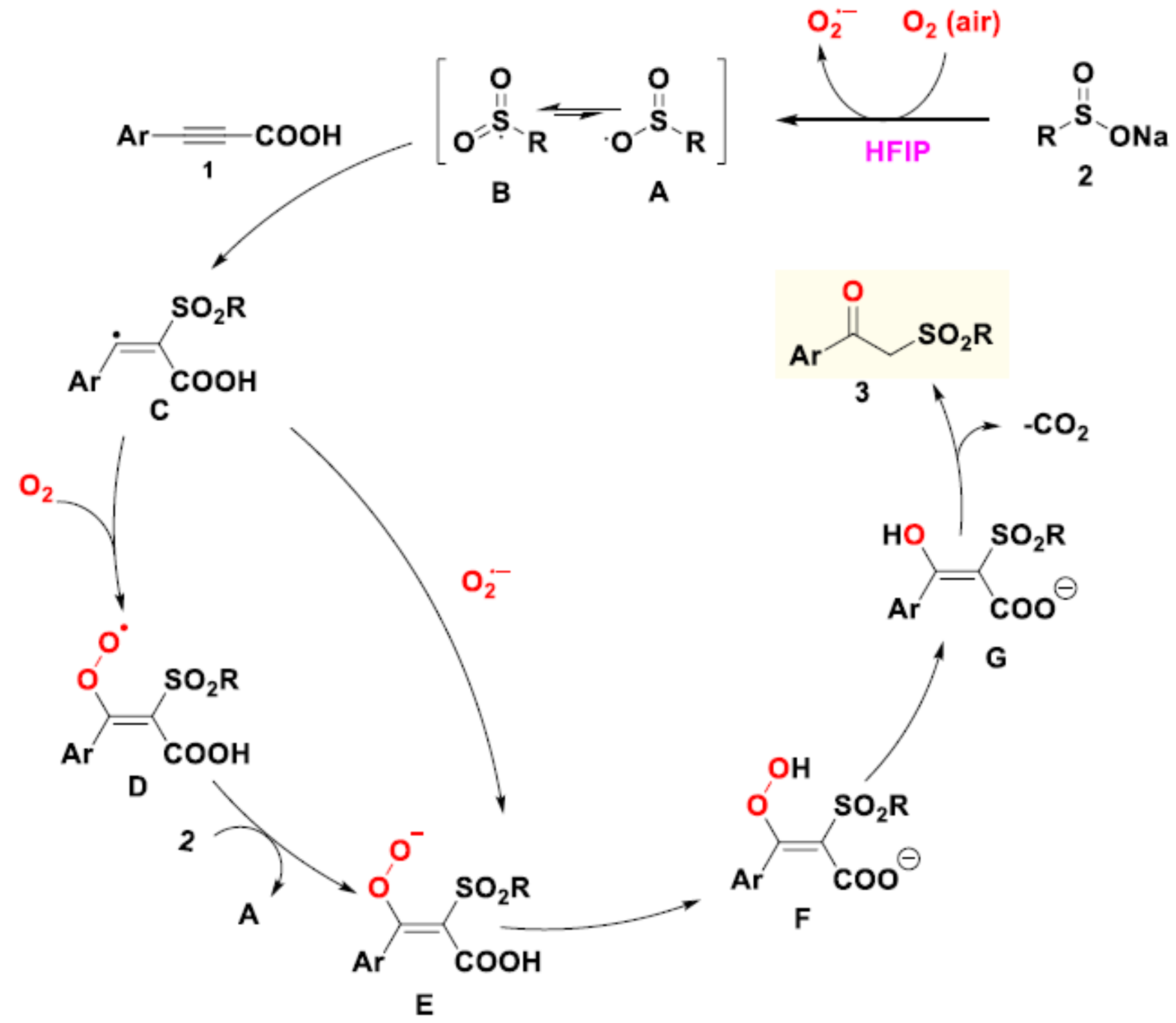

Figure 4

Plausible mechanism.

\section{Supplementary Files}

This is a list of supplementary files associated with this preprint. Click to download.

- Supportinglnformation.pdf 